\title{
MedienPädagogik
}

Zeitschrift für Theorie und Praxis der Medienbildung

Themenheft Nr. 33: Medienpädagogik und Didaktik der Informatik.

Eine Momentaufnahme disziplinärer Bezüge und schulpraktischer Entwicklungen. Herausgegeben von Torsten Brinda, Ira Diethelm, Sven Kommer und Klaus Rummler

\section{Kritische Entgegnung auf die Studie «Zur Relevanz informatischer Bildung in der Schule für den Er- werb computer- bzw. informationsbezogener Kom- petenzen»}

Torsten Brinda, Ira Diethelm, Lutz Hellmig, Johannes Magenheim, Ralf Romeike und Ulrik Schroeder

\section{Zusammenfassung}

Dieser Beitrag nimmt Bezug auf den Artikel "Zur Relevanz informatischer Bildung in der Schule für den Erwerb computer- und informationsbezogener Kompetenzen als Teilaspekt von Medienbildung", der an gleicher Stelle in einem früheren Heft erschien. Basierend auf Sekundäranalysen der ICILS 2013-Daten wird darin geschlussfolgert, es gebe einen Zusammenhang zwischen der Belegung von Informatikunterricht in der Schule und geringeren computer- bzw. informationsbezogenen Kompetenzen. Der vorliegende Beitrag legt dar, warum diese und andere Schlussfolgerungen basierend auf dem zur Verfügung stehenden Datenmaterial und der von den Autorinnen gewählten Auswertungsmethodik wissenschaftlich nicht haltbar sind.

Critical Response to the Study «On the Relevance of IT Education at School for the Purpose of Acquiring Computer and Information Literacy as part of Media Education»

\begin{abstract}
This article refers to the article "On the Relevance of IT Education at School for the Purpose of Acquiring Computer and Information Literacy as part of Media Education", which was published in an earlier issue of this journal. Based on secondary analyses of the ICILS 2013 data, its authors concluded for Germany and Switzerland that there is a relation between taking «computer science» at school and lower computer and/or information literacy. The article at hand shows why these and other conclusions are scientifically unsustainable based on the available data and the evaluation methodology chosen by the authors.
\end{abstract}




\section{Einleitung}

Dieser Beitrag nimmt Bezug auf den Artikel «Zur Relevanz informatischer Bildung in der Schule für den Erwerb computer- und informationsbezogener Kompetenzen als Teilaspekt von Medienbildung» von Birgit Eickelmann und Kerstin Drossel (2016), der im Themenheft 25 dieser Zeitschrift erschien. In diesem Beitrag verwenden die Autorinnen die Daten der ersten International Computer and Information Literacy Studie - ICILS 2013 (IEA o.J.), um im Rahmen von Sekundäranalysen der Frage nachzugehen, inwieweit ein Zusammenhang zwischen der Belegung des Faches «Informatik» durch Schülerinnen und Schüler und dem in der ICILS-Studie erhobenen Konstrukt der computer- und informationsbezogenen Kompetenz (Bos et al. 2014) besteht. Die Daten wurden an 136 allgemeinbildenden Schulen unterschiedlicher Schultypen in allen Bundesländern Deutschlands und Schweizerischen Kantonen erhoben. Je Schule liegen Datensätze von bis zu 20 zufällig aus der jeweiligen Jahrgangsstufe 8 ausgewählten Teilnehmenden vor. Im Ergebnis berichten die Autorinnen basierend auf den o.g. Daten, dass sich für Deutschland und die Schweiz zeige, dass «Schülerinnen und Schüler, die das Fach Informatik belegen, im Mittel deutlich geringere computer- und informationsbezogene Kompetenzen erreichen als die gleichaltrige Gruppe, die das Fach nicht belegt» (Eickelmann \& Drossel 2016, 80). Diese Schlussfolgerung ist basierend auf der von den Autorinnen gewählten Methode nicht haltbar, wie nachfolgend ausgeführt wird.

\section{Zur Datenerhebung}

Grundlage der Erhebung der Belegung von Informatikunterricht war ausschliesslich das Item (vgl. IEA o. J., deutschsprachige Übersetzung von den Autorinnen per Mail auf Anfrage erhalten):

«Wie häufig nutzt du während des Unterrichts einen Computer in den folgenden Fächern oder Fachbereichen? [Informatik, Informationstechnischer Unterricht o.ä.]. Als Antwortmöglichkeiten stehen die Optionen nie, in einigen Unterrichtsstunden, in den meisten Unterrichtsstunden, in jeder bzw. fast jeder Unterrichtsstunde und Ich belege dieses Fach bzw. diese Fächer nicht zur Verfügung.»

Tabelle 1 zeigt eine mittels SPSS erzeugte Auswertung der zu dieser Variable vorliegenden Daten: 
Your use of computers and the internet/How often use computers during lessons/[Information technology, computer studies]

\begin{tabular}{|c|c|c|c|c|c|}
\hline & & & & & \\
\hline & & Häufigkeit & Prozent & $\begin{array}{l}\text { Gültige } \\
\text { Prozente }\end{array}$ & $\begin{array}{l}\text { Kumulierte } \\
\text { Prozente }\end{array}$ \\
\hline \multirow[t]{6}{*}{ Gültig } & Never & 595 & 26,7 & 28,5 & 28,5 \\
\hline & In some lessons & 210 & 9,4 & 10,1 & 38,6 \\
\hline & In most lessons & 99 & 4,4 & 4,7 & 43,3 \\
\hline & In every or almost every lesson & 553 & 24,9 & 26,5 & 69,8 \\
\hline & $\begin{array}{l}\text { I don't study this subject/these } \\
\text { subjects }\end{array}$ & 629 & 28,3 & 30,2 & 100 \\
\hline & Gesamt & 2086 & 93,8 & 100,0 & \\
\hline \multirow[t]{3}{*}{ Fehlend } & $\begin{array}{l}\text { Not administered/missing by } \\
\text { design }\end{array}$ & 111 & 5,0 & & \\
\hline & $\begin{array}{l}\text { Presented but not answered/ } \\
\text { invalid }\end{array}$ & 28 & 1,3 & & \\
\hline & Gesamt & 139 & 6,2 & & \\
\hline Gesamt & & 2225 & 100,0 & & \\
\hline
\end{tabular}

Tab. 1.: Auswertung zum ICILS 2013-Item zur Computernutzung im Informatik- bzw. informationstechnischen Unterricht in Deutschland.

Fehlende Daten wurden von den Autorinnen in der weiteren Auswertung nicht berücksichtigt. Ein eigenes Item, das explizit die Belegung entsprechenden Fachunterrichts erfragte, wurde nicht verwendet. Die Ausführungen zur Datenerhebung im Beitrag von Eickelmann und Drossel (2016, 90-91) lassen das allerdings offen:

«Hinsichtlich der fachspezifischen Nutzung konnten die Schülerinnen und Schüler in allen beteiligten Bildungssystemen für verschiedene Fächer auswählen, ob sie nie, in einigen Unterrichtsstunden, in den meisten Unterrichtsstunden oder in jeder bzw. fast jeder Unterrichtsstunde einen Computer im Unterricht nutzen. Jugendliche, die ein Unterrichtsfach nicht belegen, konnten dies ebenfalls kenntlich machen.»

\section{Zur Belegung von Informatikunterricht}

Die Autorinnen schlussfolgern nun, dass alle diejenigen Schülerinnen und Schüler, die auf dem Fragebogen nicht angekreuzt haben, dass sie ein Fach des Bereichs «Informatik, Informationstechnischer Unterricht o.ä.» nicht belegen, somit also eine der ersten vier Antwortoptionen wählten, zum Befragungszeitpunkt Informatikunterricht belegen. Dieser Schluss ist in mehrerlei Hinsicht unzulässig. 
Die Fragestellung des Items bezieht sich nicht auf Belegung von Fachunterricht.

Die o.g. Fragestellung bezieht sich explizit auf die Computernutzung im Bereich «Informatik, Informationstechnischer Unterricht o.ä.». Die Antwortmöglichkeiten auf die gestellte Frage lauten somit eigentlich nur nie, in einigen Unterrichtsstunden, in den meisten Unterrichtsstunden und in jeder bzw. fast jeder Unterrichtsstunde. Weiterhin wird die Frage «Belegst Du dieses Fach bzw. diese Fächer?» beantwortet, allerdings ohne in der Fragestellung aufgeführt worden zu sein. Es kann daher nicht ausgeschlossen werden, dass an der Befragung teilnehmende Personen nicht alle Antwortalternativen bis zum Ende gelesen und, weil sie ein entsprechendes Fach bspw. nicht belegten, die Antwortoption «nie» oder ihre diesbezügliche Vermutung zur Computernutzung als Antwort gewählt haben. Die Reihenfolge der Antworten ist im Hinblick auf das dem Paper zugrundeliegenden Untersuchungsziel mindestens unlogisch. Der üblichen Praxis (z.B. Payne 1980, 102 ff.) entspräche es, zunächst eine Filterfrage nach der Fachbelegung zu stellen, um dann die mit «ja» antwortenden Probanden nach der Computernutzung in diesem Bereich zu befragen. Die Interpretation des Komplements zur Nichtbelegung als tatsächliche Belegung eines Fachs des genannten Bereichs muss daher als spekulativ bewertet werden. Die Daten haben insofern kaum Aussagekraft.

Das Komplement zur Nichtbelegung von «Informatik, Informationstechnischer Unterricht o. ä.» ist nicht «Belegung von Informatikunterricht»

Die Daten zum Item erlauben keinerlei spezifische Analyse zu Informatikunterricht, wie die Ausführungen der Autorinnen suggerieren. Da sich das Item auf die Computernutzung im Bereich «Informatik, Informationstechnischer Unterricht o.ä.» bezieht, ist eine Auswertung nur bezogen auf diesen Bereich in Gänze wissenschaftlich zulässig (unter Berücksichtigung der Kritik hinsichtlich der grundsätzlichen Interpretierbarkeit der Antworten, wie oben ausgeführt). Das Kernproblem liegt hierbei darin, dass die Autorinnen den Begriff «Informatikunterricht» als Oberbegriff für das von ihnen adressierte Feld «Informatik, Informationstechnischen Unterricht o.ä.» verwenden. Hubwieser (2007, 43-50) fasst bspw. die Bereiche «Unterstützung von Lernprozessen», "Bedienerschulung» und «Informatikunterricht» als Facetten von «informatischer Bildung» zusammen, definiert Informatikunterricht damit als Teil eines weiteren Feldes. Den inhaltlichen Schwerpunkt von Informatikunterricht charakterisiert er als «Prinzipien, Konzepte und Strategien zur Planung, Konstruktion, Beschreibung und Bewertung abstrakter Informatiksysteme» (ebd., 48), um dann auch darauf hinzuweisen: «Leider wird im schulischen Umfeld der Begriff Informatik häufig für jede Art der Beschäftigung mit dem Computer missbraucht.» Um diesem Problem zu begegnen, wurden verschiedene Facetten des Einsatzes von und der Auseinandersetzung mit Informatiksystemen im Schulunterricht in der Dagstuhl-Erklärung 
(Brinda et al. 2016) unter dem Oberbegriff «Bildung in der digitalen vernetzten Welt» zusammengefasst.

Basierend auf den erhobenen Daten und durch Erweiterung des abgefragten Bereichs durch «o.ä.» sind keinerlei spezifische Aussagen möglich, auf welche unterrichtlichen Erscheinungsformen sich die Antworten der Teilnehmenden beziehen. Denkbar wäre hier neben tatsächlichem Informatikunterricht in Wahl-, Wahlpflichtoder Pflichtform die Bezugnahme auf fächerintegrierte Medienbildungskonzepte, Konzepte informationstechnischer Grundbildung, Arbeitsgruppen (AGs) oder jedwede andere Form von organisierter Computernutzung. Weiterhin ist völlig offen, in welchem Umfang sich etwaige Belegungen unter den Teilnehmenden auf die o.g. Erscheinungsformen verteilen. All diese Erscheinungsformen pauschal unter den Formulierungen «Informatikunterricht» oder «Fach Informatik» zu subsumieren entspricht nicht der wissenschaftlichen Begriffsbildung und ist daher irreführend.

Es ist völlig offen, ob sich die Angaben der Teilnehmenden auf curriculumskonformen Unterricht von ausgebildeten Fachlehrkräften beziehen und, wenn ja, welche Curricula dem zugrunde liegen.

Wollte man Zusammenhänge mit Informatikunterricht untersuchen, wäre es erforderlich, sicherzustellen, dass alle Teilnehmenden die gleichen Chancen auf den Erwerb entsprechender Kompetenzen im Informatikunterricht gehabt hätten. Das ist im vorliegenden Fall in mindestens dreierlei Hinsicht nicht gegeben:

a) Die Verankerung der Erscheinungsformen Informatik als Schulfach, informationstechnische Grundbildung, Medienbildungskonzepte oder Informatik- oder MedienAGs variiert erheblich zwischen Bundesländern, Kantonen und Schulformen, verpflichtende Informatikangebote gibt es nur an wenigen Stellen (vgl. Starrus 2010). Es kann also nicht ausgeschlossen werden, dass die Teilnehmenden zum Erhebungszeitpunkt entweder curricular oder durch den Unterricht der jeweiligen Lehrkraft bedingt noch gar keine Gelegenheiten zum Erwerb der überprüften Kompetenzen hatten.

b) Über den zeitlichen Gesamtumfang an besuchtem Unterricht des zur Rede stehenden Bereichs liegen keinerlei Informationen vor. Wenige Unterrichtsstunden werden gemäss der Interpretation der Autorinnen gleichgesetzt mit mehrjährigem Fachunterricht. Ist eine entsprechende Differenzierung für die Untersuchung nicht von Belang, so ist daraus zu folgern, dass der Erwerb der untersuchten Kompetenzen offenbar unabhängig von der darauf investierten Unterrichtszeit ist, dass also in wenigen Unterrichtsstunden dieselbe Kompetenzentwicklung möglich ist, wie an anderer Stelle nach mehreren Jahren. Das darf im Hinblick auf die untersuchten informations- und computerbezogenen Kompetenzen als höchst fragwürdig bezeichnet werden. 
c) Weiterhin liegen keinerlei offizielle Angaben darüber vor, in welchem Umfang entsprechender Unterricht von dazu fachlich qualifizierten Lehrkräften durchgeführt wird und es kann somit nur darüber spekuliert werden, wie die Qualifikationssituation der Lehrkräfte war, auf deren Unterricht sich die Angaben der Teilnehmenden beziehen.

Die beteiligten Schülerinnen und Schüler hatten insofern höchst unterschiedliche Chancen, in Unterricht im o. g. Bereich die im Rahmen der Studie überprüften Kompetenzen zu erwerben oder zu festigen. Jedwede pauschale Herstellung eines Zusammenhangs mit Unterricht aus diesem Bereich lässt alle diese Aspekte ausser Acht, hat daher keinerlei Aussagekraft von wissenschaftlichem Belang.

\section{Die Berücksichtigung von Belegungen in früheren Jahrgangsstufen ist völlig unklar.} Die Antwortoption «Ich belege dieses Fach bzw. diese Fächer nicht» deutet darauf hin, dass es um aktuelle Belegungen geht, also Unterricht in der Jahrgangsstufe 8. Ob das den an der Studie teilnehmenden Personen in allen Fällen klar war, oder ob auch Belegungen in der Vergangenheit zur Beantwortung der Computernutzung herangezogen wurden, kann anhand der Daten nicht geklärt werden. Das führt allerdings zu einem weiteren Problem: Personen, die vor der Jahrgangsstufe 8 Informatik belegten, wie bspw. alle Schülerinnen und Schüler der Jahrgangsstufe 6 und 7 am Bayerischen Gymnasium im Fach Natur und Technik/Schwerpunkt Informatik, und die die Belegung bezogen auf Jahrgangsstufe 8 (kein Informatikunterricht in Jahrgangsstufe 8 am Bayerischen Gymnasium, vgl. ISB o.J.) wahrheitsgemäss verneinten, würden gemäss der Interpretation der Autorinnen zur Gruppe der Informatik-Nichtbeleger gezählt, trotz erheblicher informatischer Vorbildung, was als erhebliche Verfälschung gewertet werden müsste. Auch dieses Problem schliesst eine Interpretierbarkeit der Daten in der von den Autorinnen dargelegten Weise aus.

\section{Zur Computernutzung im «Informatikunterricht»}

Basierend auf der o.g. Erhebung der Belegung von «Informatikunterricht» werten die Autorinnen dann auch die Computernutzung in diesem Unterricht aus: «Für das Fach Informatik liegen die Nutzungshäufigkeiten höher, aber sogar hier geben nur 41,7 Prozent der Achtklässlerinnen und Achtklässler, die das Fach belegen, an, nie Computer im Unterricht zu nutzen. In der Schweizer Stichprobe liegt die Nutzungshäufigkeit von Computern durch Schülerinnen und Schüler in der Schule nur geringfügig über dem Ergebnis für Deutschland.» (Eickelmann \& Drossel, 88-89). Bzgl. dieses Ergebnisses ist folgende weiterführende Analyse auf den veröffentlichten Daten interessant: Es gibt im veröffentlichten Datensatz in fast jeder teilnehmenden Schule (in 92\% 
aller teilnehmenden Schulen) mindestens eine/n Schüler/in, der bzw. die angibt, im abgefragten Unterrichtsbereich nie mit dem Computer zu arbeiten. Andererseits gibt es an $74 \%$ der Schulen mindestens eine/n Schüler/in, der bzw. die angibt, fast immer am Computer zu arbeiten. Diese Zahlen widersprechen jedweder Plausibilität - auch im Hinblick auf die o. g. Definition von Informatikunterricht nach Hubwieser (2007).

Da bislang keine nationalen Studien zur Beschreibung entsprechenden Fachunterrichts vorliegen, kann nicht ausgeschlossen werden, dass in unbekanntem Umfang tatsächlich auch reiner Tafel- und Kreideunterricht stattfindet. Die erhebliche Variation von Antworten zur Computernutzung innerhalb der Schulen könnte auf verschiedene Erklärungsmodelle zurückgeführt werden, z.B.

- Es könnte von unterschiedlichem Unterricht innerhalb derselben Schule berichtet werden.

- Die Ursache könnte in Ausstattungsproblemen und/oder schlecht organisierter Rechnerarbeit liegen. Wenn weniger Geräte zur Verfügung stehen, als Lernende am Unterricht teilnehmen und dominante Schülerinnen und Schüler die Arbeit am Gerät in die Hand nehmen, könnte es vorkommen, dass andere nur daneben sitzen.

- Teilnehmende könnten irrtümlich die Option «nie» gewählt haben, da sie entsprechenden Fachunterricht nicht belegen, insofern auch keinen Computer darin nutzen.

- In Jahrgangsstufe 8 könnte entsprechender Unterricht zum Befragungszeitpunkt gerade erst begonnen haben, so dass noch kein Rechnereinsatz stattfand.

Die undifferenzierte Angabe einer Gesamtquote von Achtklässlerinnen und Achtklässlern, die nie einen Computer im entsprechenden Fachunterricht nutzen, wird den potenziell vielfältigen Ursachen nicht gerecht und lässt daher keine fundierten Aussagen zu.

\section{Zu den Belegzahlen und Kompetenzunterschieden}

Bzgl. der «Informatikbelegung» in ihrer Stichprobe geben die Autorinnen eine Belegungsquote von $80,4 \%$ für die Schweiz und $71,4 \%$ für Deutschland an. Diese Zahlen widersprechen den verfügbaren statistischen Angaben der Kultusministerien und der Kultusministerkonferenz. Im Rahmen der Erarbeitung des vorliegenden Textes wurden im November 2016 alle Kultusministerien der Länder in Deutschland per EMail angeschrieben mit der Frage, welcher Anteil der deutschen Schülerinnen und Schüler in der Jahrgangsstufe 8 mit Informatikunterricht (Pflicht-, Wahlpflicht- oder Wahlfach) an allgemeinbildenden Schulen erreicht wird. 
Diese Frage wurde auch an das Sekretariat der Kultusministerkonferenz gerichtet. Für die Sekundarstufe I liegen bei der KMK It. Antwort per E-Mail keine bundesweiten statistischen Daten vor. In der Sekundarstufe /I wurde das Fach Informatik im Schuljahr 2014/15 in Deutschland als Grund- oder Leistungskurs von in Summe $16,96 \%$ aller Schülerinnen und Schüler gewählt, von 15,98\% als Grundkurs und $0,98 \%$ als Leistungskurs.

In Baden-Württemberg belegten laut Auskunft des dortigen Kultusministeriums im Schuljahr 2015/16 5,17\% aller Schülerinnen und Schüler von Werkreal-/ Hauptschule, Realschule, allgemeinbildendem Gymnasium, Gemeinschaftsschule Sek. I und Schulen besonderer Art ein Wahlpflichtfach Informatik. Arbeitsgemeinschaften (Informatik / Datenverarbeitung und Datenverarbeitung / Informationstechnik) werden an den genannten Schultypen in der Sek. I insgesamt zu 2,41\% belegt.

In Hessen nahmen im Schuljahr 2015/16 von den Schülerinnen und Schülern ohne Migrationshintergrund 3,1\% an Förderschulen, 9,8\% an Grund-Haupt-Realschulen, 7,9\% an Gymnasien, 6,2\% an Schulformübergreifenden Gesamtschulen und 11,1\% an Schulformbezogenen Gesamtschulen laut Auskunft des Hessischen Kultusministeriums an Pflicht-, Wahlpflicht oder Wahlunterricht im Fach Informatik teil.

In Nordrhein-Westfalen wurden laut der offiziellen Schulstatistik (MSWNW 2016) an den Schulformen Haupt-, Real-, Sekundar-, Gemeinschafts- und Gesamtschule, Gymnasium sowie Förderschule im Schuljahr 2015/16 in Jahrgangsstufe 8 insg. 20,9\% der Schülerinnen und Schüler mit Informatikunterricht erreicht.

Sachsen hat verpflichtenden Informatikunterricht implementiert: alle Schülerinnen und Schüler an Förderschulen mit abschlussorientiertem Unterricht, an Mittel-/ Oberschulen und Gymnasien belegten It. Sächsischem Kultusministerium im Schuljahr 2016/17 eine Stunde verpflichtenden Informatikunterricht, 4\% der o.g. Schülerinnen und Schüler zusätzlich einen zweistündigen Wahlpflichtunterricht in Informatik und Medien (an Mittel-/Oberschulen).

Aus den Ländern Bremen, Rheinland-Pfalz, Niedersachsen, Thüringen erfolgte die Meldung, dass entsprechende Daten dort nicht in der angefragten Weise verfügbar seien. Die übrigen Kultusministerien antworteten bis zum Zeitpunkt der Niederschrift dieses Beitrags auf die Anfrage nicht.

Es gibt keinerlei Indiz für die Annahme, dass sich in den übrigen Bundesländern, die kein Informatikpflichtfach implementiert haben, ein völlig anderes Bild bzgl. der Belegzahlen zeigen könnte. Unabhängig von allen bereits dargelegten Problemen bzgl. der Interpretierbarkeit müsste ausgehend von den in der Stichprobe ermittelten Belegungsquoten von Informatikunterricht (80,4\% für die Schweiz und $71,4 \%$ für Deutschland) somit deren Repräsentativität in Frage gestellt werden.

Die undifferenzierte Angabe einer Gesamtquote von Achtklässlerinnen und Achtklässlern, die nie einen Computer im entsprechenden Fachunterricht nutzen, wird den potenziell vielfältigen Ursachen nicht gerecht und lässt daher keine fundierten Aussagen zu. 


\section{Fazit}

Die dargelegten Probleme führen zu der Schlussfolgerung, dass die Aussage, dass sich für Deutschland und die Schweiz zeige, dass «Schülerinnen und Schüler, die das Fach Informatik belegen, im Mittel deutlich geringere computer- und informationsbezogene Kompetenzen erreichen als die gleichaltrige Gruppe, die das Fach nicht belegt» (Eickelmann \& Drossel 2016, 80) wissenschaftlich nicht haltbar ist. Die Autorinnen geben selbst an, dass überproportionale Belegungen durch Schüler, die bei der ICILS 2013 durchweg schlechter abschnitten, als Schülerinnen, ein plausibles Erklärungsmodell sein könnten (ebd., 104).

Zum Teilergebnis, dass die Jugendlichen, die «Informatik» belegen und im Informatikunterricht (oder einem Fach zur informatischen Bildung) einen Computer benutzen, im Mittel bessere Ergebnisse erzielten, als die Vergleichsgruppe (ebd., 80), ist festzuhalten, dass darunter curriculumskonformer Informatikunterricht durch ausgebildete Fachlehrkräften fallen dürfte - das Modell, das von Befürwortern des Faches gefordert wird. In einer Zeit, in der sehr intensiv darum geworben und gerungen wird, informatische Kompetenzen verbindlicher in den Curricula der allgemeinbildenden Schulen zu verankern, birgt ein Beitrag, der «Informatikbelegung» pauschal mit «im Mittel deutlich geringeren computer- und informationsbezogene Kompetenzen» in Zusammenhang bringt, vor dem Hintergrund der geschilderten Probleme bildungspolitische Brisanz und könnte von Kritikern informatischer Bildung leicht für deren Position instrumentalisiert werden, da die ICILS 2013 eine international beachtete und gewürdigte Studie war und ist. Wenngleich die Erkenntnisse der ICILSStudie insgesamt damit in keinster Weise herabgewürdigt werden sollen, müssen die Ergebnisse des hier zur Rede stehenden Beitrags deshalb entschieden zurückgewiesen werden. Insgesamt zeigt sich, dass es noch grossen Bedarf zur Erforschung der informatischen Bildung in Deutschland gibt.

\section{Literatur}

Bos, Wilfried, Birgit Eickelmann, Julia Gerick, Frank Goldhammer, Heike Schaumburg, Knut Schwippert, Martin Senkbeil, Renate Schulz-Zander, und Heike Wendt, Hrsg. 2014. ICILS 2013: computer- und informationsbezogene Kompetenzen von Schülerinnen und Schülern in der 8. Jahrgangsstufe im internationalen Vergleich. Münster: Waxmann.

Brinda, Torsten, Ira Diethelm, Rainer Gemulla, Ralf Romeike, Johannes Schöning, Carsten Schulte, und et al. 2016. Dagstuhl-Erklärung: Bildung in der digitalen vernetzten Welt. Zugriff 20.01.2017. https://www.gi.de/aktuelles/meldungen/detailansicht/article/dagstuhlerklaerung-bildung-in-der-digitalen-vernetzten-welt.html. 
Eickelmann, Birgit, und Kerstin Drossel. 2016. Zur Relevanz informatischer Bildung in der Schule für den Erwerb computer- und informationsbezogener Kompetenzen als Teilaspekt von Medienbildung. MedienPädagogik: Zeitschrift für Theorie und Praxis der Medienbildung 25, 80-108. https://doi.org/10.21240/mpaed/25/2016.10.29.X.

Hubwieser, Peter. 2007. Didaktik der Informatik. 3. Auflage. Berlin: Springer.

International Association for the Evaluation of Educational Achievement (IEA) (o. J.). Student background data (ICILS 2013, Germany). http://rms.iea-dpc.org.

Staatsinstitut für Schulqualität und Bildungsforschung des Bundeslandes Bayern - ISB (o.J.): Lehrplan des achtjährigen Gymnasiums. http://www.isb-gym8-lehrplan.de/.

Ministerium für Schule und Weiterbildung des Landes Nordrhein-Westfalen - MSWNW. 2016. Das Schulwesen in Nordrhein-Westfalen aus quantitativer Sicht 2015/16. 1. Auflage. https:// www.schulministerium.nrw.de/docs/bp/Ministerium/Service/Schulstatistik/AmtlicheSchuldaten/Quantita_2015.pdf.

Payne, Stanley Le Baron. 1980. The Art of Asking Questions: Studies in Public Opinion, 3. Princeton, NJ: Princeton University Press.

Starrus, Isabelle. 2010. Synopse zum Informatikunterricht in Deutschland. Analyse der informatischen Bildung an allgemeinbildenden Schulen auf der Basis der im Jahr 2010 gültigen Lehrpläne und Richtlinien. Bachelor-Arbeit. Dresden: TU Dresden. http://dil.inf.tu-dresden.de/ uploads/media/Bakkalaureatsarbeit_Isabelle_Starruss_01.pdf. 